\title{
Modeling Polycrystals with Regular Polyhedra
}

\author{
Paulo Rangel Rios ${ }^{\mathrm{a} *}$, Martin Eden Glicksman ${ }^{\mathrm{b} *}$ \\ ${ }^{a}$ Universidade Federal Fluminense, Escola de Engenharia Industrial Metalúrgica de \\ Volta Redonda, Av. dos Trabalhadores, 420, 27255-125 Volta Redonda - RJ, Brazil \\ ${ }^{\mathrm{b}}$ Rensselaer Polytechnic Institute, Materials Science \& Engineering Department \\ Troy, NY 12180-3590 USA
}

Received: October 29, 2005; Revised: February 6, 2006

\begin{abstract}
Polycrystalline structure is of paramount importance to materials science and engineering. It provides an important example of a space-filling irregular network structure that also occurs in foams as well as in certain biological tissues. Therefore, seeking an accurate description of the characteristics of polycrystals is of fundamental importance. Recently, one of the authors (MEG) published a paper in which a method was devised of representation of irregular networks by regular polyhedra with curved faces. In Glicksman's method a whole class of irregular polyhedra with a given number of faces, $N$, is represented by a single symmetrical polyhedron with $N$ curved faces. This paper briefly describes the topological and metric properties of these special polyhedra. They are then applied to two important problems of irregular networks: the dimensionless energy 'cost' of irregular networks, and the derivation of a 3D analogue of the von Neumann-Mullins equation for the growth rate of grains in a polycrystal.
\end{abstract}

Keywords: microstructure, grain growth, analytical methods, foams, polycrystals

\section{Introduction}

The polycrystalline structure is of paramount importance to materials science and engineering. It is an example of a space-filling irregular network structure that also occurs in foams as well as in certain biological tissues. The technological importance of polycrystals derives from the fact that the majority of industrial crystalline materials are used as polycrystals.

Therefore, accurate description of the characteristics of polycrystals is of fundamental importance. A complete description of such a network involves the knowledge of the geometric characteristics of the individual crystals, of their crystallographic orientation, i.e., their crystallographic texture, of the nature of the interfaces between individual crystals, among others. Not only is it important to know these characteristics at one instance in time, but also to predict their dynamic behavior when such structures change as a function of time because of grain growth.

Nevertheless, a purely geometric characterization of an irregular network of grains is not easy. One needs to know the volume and shape of the individual grains. This requires tedious and time-consuming experimental techniques such as serial sectioning, or even applying more demanding methods such as "disintegrating" the polycrystal, for example, by adding gallium to an aluminium polycrystal and inspecting the individual grains. In practice, one seldom does this, and, instead, often a single measurement is used: one determines just the "grain size" using either the ASTM number or the mean intercept length. Even if detailed measurements were available, making sense of a variety of irregular polyhedra would still be a daunting task.

Recently, one of the authors (MEG) has published a paper in which a method of representation of irregular networks by regular polyhedra with curved faces was devised ${ }^{1}$. In Glicksman's method a whole class of irregular polyhedra with a given number of faces, $N$, is represented by a single symmetrical polyhedron with $N$ faces. These polyhedra are 'regular polyhedra' with curved faces, constructed in such a way that they satisfy the average topological constraints imposed by a space filling network. Glicksman called these special regular polyhedra with curved faces 'average $N$-hedra' or ANHs. In this work they will be called "ANHs", for brevity. This approach significantly simplifies the mathematical treatment of irregular networks. The geometric properties of the ANHs can be calculated exactly, as closed-form expressions are available for all their important geometric and kinetic properties. The ANHs act as "proxies" for analyzing irregular network grains, allowing rigorous treatment of several longstanding problems $s^{2-5}$ pertaining to these networks.

This paper briefly describes the topological and metric properties of the ANHs. The ANH concept is then applied to two important problems of irregular networks: the dimensionless energy 'cost' of irregular networks (in essence their "stored" free energy) and the derivation of a 3D analogue of the von Neumann-Mullins equation, to predict the growth rates of polycrystals. This paper will focus on the properties of ANHs rather than on the detailed mathematical derivations that can be found elsewhere ${ }^{1-5}$.

\section{Construction of ANHs}

A polycrystalline structure is not just an aggregate of contiguous polyhedra. The grain boundary energies or surface tensions impose certain topological constraints on the network polyhedra. These 'network' conditions must be satisfied by the ANHs as well, if they represent the average behavior of space-filling grains.

Any polyhedron must satisfy Euler's formula relating number of edges, $E$, vertices, $V$ and faces, $N$ :

$$
N-E+V=2
$$

In a grain network, three faces always meet along a common edge, and four faces always meet at a common vertex. For an isolated polyhedron this is equivalent to requiring that two polyhedral faces meet along a common edge, and that three edges meet at a vertex. 
Smith ${ }^{6}$ found an expression for the number of edges per face, $p$, for a polyhedron having trihedral vertices:

$$
p=6-\frac{12}{N}
$$

Moreover, equilibrium conditions require that the three faces meet with dihedral angles equal to $2 \pi / 3=120^{\circ}$, and the edges meet at the common vertex with the tetrahedral angle equal to ar$\cos (-1 / 3) \cong 109.47^{\circ}$. These conditions are known as "Plateau rules," which are overly restrictive, inasmuch as the polycrystal must be in full equilibrium at every edge and vertex. A much more general, and far less restrictive, condition is to require that the three faces meet with an average dihedral angle equal to $2 \pi / 3=120^{\circ}$ and edges meet with the average tetrahedral angle equal to $\operatorname{arcos}(-1 / 3) \cong 109.47^{\circ}$. These average network conditions are obeyed by ANHs. The construction of an ANH may be exemplified by constructing a 4-hedron. The starting point for the construction of a symmetrical curved-face 4-hedron is a regular polyhedron with four flat faces: i.e., the regular tetrahedron. In order to make the tetrahedron satisfy the average network constraints the flat faces must be curved into spherical caps. When this is done the edges must also be curved. Figure 1 shows a schematic drawing of this 'curved-face tetrahedron'. The angles between the normals at the center of the faces, $\alpha$, remain the same after they have been curved, but the angle between normals adjacent to the edges are now smaller than $\alpha$. If the faces are sufficiently curved, then this angle can be made to be equal to $60^{\circ}$, corresponding to an interior dihedral angle equal to $120^{\circ}$. It is also necessary to curve the edges so that they meet at the common vertex with the correct tetrahedral angle, $109.47^{\circ}$.

This process can be repeated for all regular flat faced polyhedra. Unfortunately, not all regular polyhedra can be constructed. The reason for this restriction can be understood using Smith's formula above. The number of edges per face, $p$, is determined by the number of faces. For a polyhedron to be constructible, $p$ must evidently be an integer number. This only happens for $N=3,4,6$ and 12, giving $p=2,3,4$ and 5 , respectively. All the constructible ANHs are illustrated in Figure 2. In Figure 2 the volumes of these ANHs are scaled so that the distance between nearest vertices, called the gauge, $\lambda$, is equal is all cases.

This example shows why the ANHs must have curved faces. The curvature of these faces is an essential property demanded by the polycrystalline network.

\section{Properties of ANHs}

Even though ANHs cannot be constructed for every value of $N$ it is nonetheless possible to find all their topological and metric properties: curvature, volume, area, edge length ${ }^{1}$. These will be summarized here.

An ANH with $N$ curved faces has 3( $N-2)$ edges and 2( $N-2)$ vertices. The radius of curvature of a face of an $\mathrm{ANH}, R$, is:

$$
R=\frac{1}{2} \csc \left(\frac{\alpha}{2}-\frac{\pi}{6}\right)\left(\cot \frac{\omega}{2} \sin \frac{\alpha}{2}-\csc \frac{\omega}{2} \sin \frac{\alpha}{2}-\cot \frac{\pi}{p}\right) \lambda
$$

where $\lambda$ is the metric gauge, or scale factor, chosen here as the distance between nearest neighbors vertices. The parameters are defined as:

- $\alpha$ is the angle between normals at the center of the faces, it is also equal to the angle between face normals of the corresponding flat faced polyhedron;

- $p$ is the average number of edges per face; and

- $\omega$ is the angle by which an edge must be curved in order that three edges meet with the tetrahedral angle of $109.47^{\circ}$.

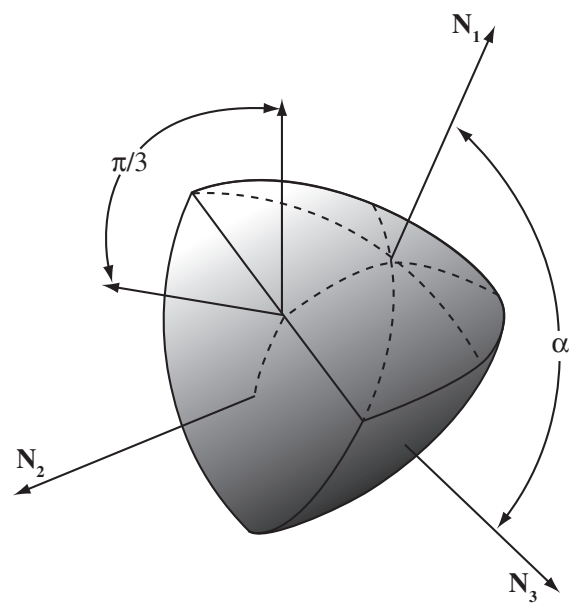

Figure 1. Geometry of the average 4-hedron. Depiction of the interfacial angle, $\alpha \approx 109.5^{\circ}$, is shown between adjacent face-centered normals, $\mathbf{N}_{1}$ and $\mathbf{N}_{2}$. Owing to the curved face the face normals rotate and meet at the edge with an angle equal to $60^{\circ}$ prescribed by equilibrium condition at the triple lines. Notice that the angle between face normals, $60^{\circ}$, is half the dihedral angle, $120^{\circ}$.
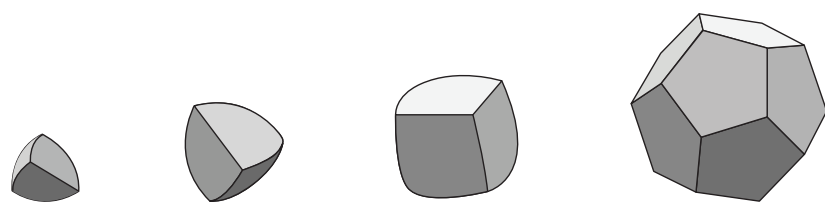

Figure 2. The four constructible ANHs of unit vertex to vertex distance. From left to right: $\mathrm{N}=3,4,6$, and 12 , with their volumes approximately proportional to $0.350,0.764,2.11$, and 9.25, respectively. Each unitary ANH in the interval $3 \leq N \leq 13$ consists of a fixed volume enclosed by $N$ identical convex curved faces intersecting at identical curved edges of unit chordal length between vertices. Edges meet three at a time at identical trihedral vertices. Vertices are symmetrically disposed about the volume centroid. All ANHs for $N \geq 14$ have concave faces, and none are constructible. $N$ :

$\alpha, p$ and $\omega$ are given below as a function of the number of faces,

$$
\begin{aligned}
& \alpha=4 \arctan \sqrt{1-2 \sec \left(\frac{\pi}{2(N-2)}\right) \cos \left(\frac{\pi(2 N-3)}{6(N-2)}\right)} \\
& p=6-\frac{12}{N} \\
& \omega=\pi+2 \arctan \left(\sin \frac{\alpha}{2} \tan \frac{\pi}{p}\right)-2 \arccos \left(-\frac{1}{3}\right)
\end{aligned}
$$

The formula for the number of edges per face, $p$, Equation 2, is repeated here for convenience.

The radius of curvature of a face allows two important quantities to be calculated: the mean curvature, $H$, and the Gaussian or total curvature, $K$. The mean curvature of an interface is the average of its two principal curvatures, $k_{1}$ and $k_{2}$. For a spherical surface of radius $R$ the principal curvatures are equal, so that:

$$
H=\frac{1}{2}\left(k_{1}+k_{2}\right)=\frac{1}{2}\left(\frac{1}{R}+\frac{1}{R}\right)=\frac{1}{R}
$$

The mean curvature is important because it is related to the interface velocity, $v$, in cases of curvature-driven interface movement by means of the well-known expression:

$$
v=-\gamma M H
$$


The minus sign applies because $H$ is positive for convex surfaces and the interface always migrates towards the curvature center on the concave side.

The Gaussian curvature of an interface is the product of its two principal curvatures. Full discussion of the fundamental significance of the Gaussian curvature is beyond the scope of this paper. For a spherical surface of radius $R$ the Gaussian curvature is:

$$
K=\frac{1}{R^{2}}
$$

The mean curvature of the face of an ANH can be better visualized in Figure 3 where it is plotted against the number of faces. For comparative purposes the scaling factor, $\lambda$, was taken to have unit value for all ANHs. The mean curvature is positive for $N<13$ and negative for $N>14$. The exact point at which $H$ changes sign is $N=N_{C} . N_{C}$ is given by:

$$
N_{C}=2+\frac{\pi}{2 \arctan \sqrt{\tan ^{3} \frac{\pi}{12}}} \cong 13.397332571438 \ldots
$$

The ANH possessing $N_{C}$ faces is called the 'critical' ANH or $N_{C}$-hedron. All faces of the $N_{C}$-hedron possess zero mean curvature; in other words, they are flat. If it were possible to construct a network just using $N_{C}$-hedra there would be no pressure difference across the flat interfaces or, equivalently, the chemical potential would remain steady throughout the polycrystal. Interface motions would cease, as the pressure difference across flat interfaces is zero. Such a network would therefore have the minimum free energy, and it would be in state of metastable equilibrium. Although the $N_{C}$-hedron is not constructible, one can calculate its properties exactly as will be shown below.

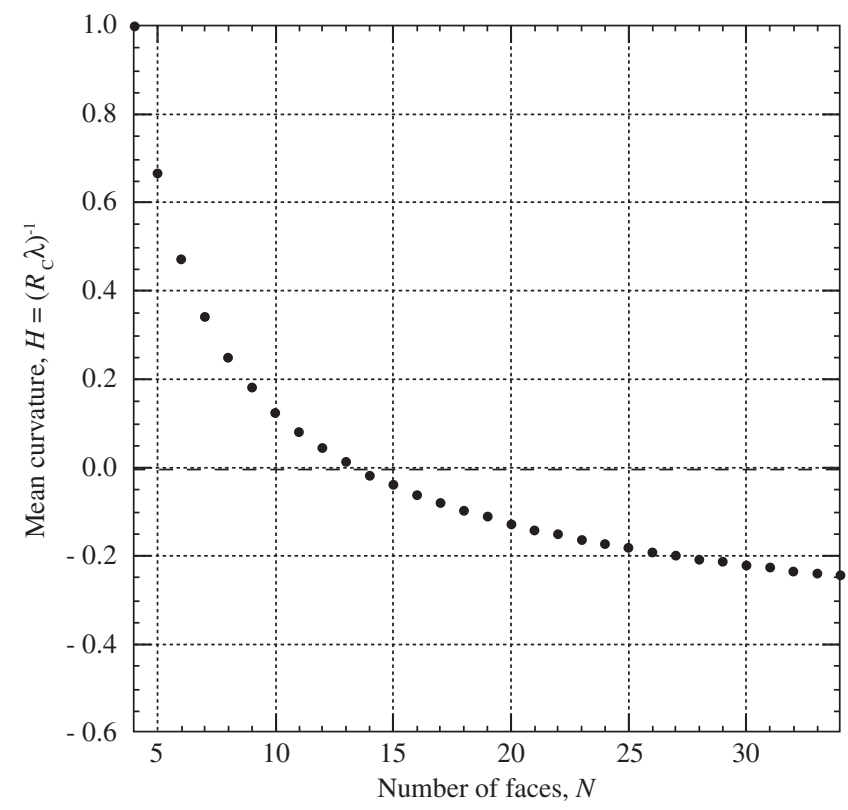

Figure 3. Mean curvature, $H=1 /(R \lambda)$ vs. number of faces on ANHs. The values of $N$ cover the practical range encountered in network structures such as polycrystals and foams. The mean curvature divides the population of ANHs: for $N \leq 13$ the mean curvatures are positive, implying shrinkage, whereas for $N \geq 14$ the mean curvatures are negative, implying growth. It is interesting to note that none of the ANH's have exactly zero mean curvature, so that in three dimensions network cells are, on average, either shrinking or growing; i.e., none are conditionally stable polyhedra.
For $N<N_{C}$ the ANHs have convex faces, see Figure 2, and their interfaces tend to migrate towards the centroid of the ANHs, so that, the ANHs tends to shrink and disappear. The opposite is true for $N>N_{C}$. The interfaces are concave and they tend to move away from the center of the ANHs and thus ANHs with $N>N_{C}$ tend to grow.

Volume, area, and edge length are other important properties of the ANHs. The area, $A$, is given by:

$$
\begin{aligned}
& A=g(N) 4 \pi R^{2} \\
& g(N)=\frac{1}{4 \pi}(4 \pi-3(N-2) \omega-2(N-2) \Omega)
\end{aligned}
$$

where $\Omega=2 \pi-3 \arccos (-1 / 3) \cong 0.551287$. The volume, $V$, is:

$$
\begin{aligned}
& V=f(N) \frac{4 \pi R^{3}}{3} \\
& f(N)=\frac{N}{2}+\frac{N-2}{16 \pi}\left(2^{\frac{3}{2}}-57 \arccos \frac{1}{3}+\right. \\
& \left.33 \arcsin \left(\frac{2}{\sqrt{3}} \cos \frac{\pi}{p}\right)-\tan \arcsin \left(\frac{2}{\sqrt{3}} \cos \frac{\pi}{p}\right)\right)
\end{aligned}
$$

The total edge length is:

$$
L=\frac{3^{\frac{3}{2}}}{2}(N-2) \omega R
$$

These expressions, however, cannot be used to find the area and volume of the critical ANH, because $R$ itself is infinite. Each of the metrical quantities listed above may be calculated by direct geometrical methods 5 :

$$
\begin{aligned}
& A_{C}=\lambda^{2} \frac{3 \pi}{2}\left(\frac{\tan \left(\frac{1}{2} \arccos \frac{1}{3}\right)}{\arctan \sqrt{\tan ^{3} \frac{\pi}{12}}}\right) \cong \lambda^{2} \times 24.1773934 \ldots \\
& V_{C}=\lambda A_{C} \frac{\cot \left(\frac{1}{2} \arccos \frac{1}{3}\right)}{6 \tan \frac{\pi}{6}} \cong \lambda^{3} \times 9.8703795 \ldots \\
& L_{C}=\lambda 3\left(N_{C}-2\right) \sqrt{\frac{4 A_{C}}{N_{C} p \cot \frac{\pi}{p}}}= \\
& \lambda 3\left(N_{C}-2\right) \cong \lambda \times 34.1919977 \ldots
\end{aligned}
$$

\section{Dimensionless Energy 'Cost' of Polycrystals}

Following Cox and Fortes ${ }^{7}$, we define a dimensionless energy cost, $G(N)$, to construct a polyhedron with $N$ faces,

$$
G(N)=\frac{1}{2} \frac{A(N)}{(V(N))^{\frac{2}{3}}}
$$

The dimensionless energy cost, Equation 15, may be interpreted as (one-half) the surface free energy required to create an isolated $\mathrm{N}$-faced polyhedron of arbitrary size scale, given that $G(N)$ is independent of the polyhedron's gauge or linear metric, $\lambda$. By summing $G(N)$ for all the polyhedral cells comprising a space-filling polycrystal, one arrives at an estimate of the dimensionless excess free energy of the grain boundary network. The sum of $G(N)$ for all polyhedral grains, divided by the number of grains, yields the average dimensionless surface energy per grain to construct the polycrystal, $\langle G\rangle$, namely,

$$
<G>=\frac{1}{n} \sum_{i=1}^{i=n} G_{i}(N)
$$


Figure 4 displays a composite plot of many dimensionless energy costs, $G, v s$. the number of faces, $N$, calculated for a variety of constructible irregular polyhedra. Areas and volumes for these (constructible) polyhedra were obtained by high-accuracy simulations performed by Cox* using Brakke's Evolver program ${ }^{8}$, and then converted to dimensionless energy costs via Equation 15 . The values calculated for all integer unitary ANHs in the range $4 \leq N \leq 34$ are also shown. The $G$-values for the ANHs were calculated on the basis of their theoretically derived areas and volumes shown in section 3 . Note the near-perfect agreement achieved between the simulated and theoretical $G$-values for the three constructible ANHs that appear on this plot, where $N=4,6$, and 12 .

Two important observations can be made from Figure 4. The first is that the range of values assumed by the dimensionless energy costs is narrow-amounting to only $2.6 \%$ of the average value. The second observation is that the $G$-values for the set of ANHs shown form a sharp lower bound to the lowest values calculated from Cox's Surface Evolver data for a selection of constructible polyhedra in each topological class. The most efficient constructible integer polyhedra fall as close as within approximately $0.1 \%$ of the dimensionless energy cost of their corresponding ANHs. In not a single instance, however, does any constructible polyhedron have a lower dimensionless energy cost than its ANH counterpart. Where the ANHs happen to be constructible, the simulation data and the analytical value agree within the numerical tolerance of the Evolver program.

As a result, one can conclude that ANHs provide both a lower bound to the energy cost and an excellent approximation to constructible irregular polyhedra. In other words, these figures are consistent

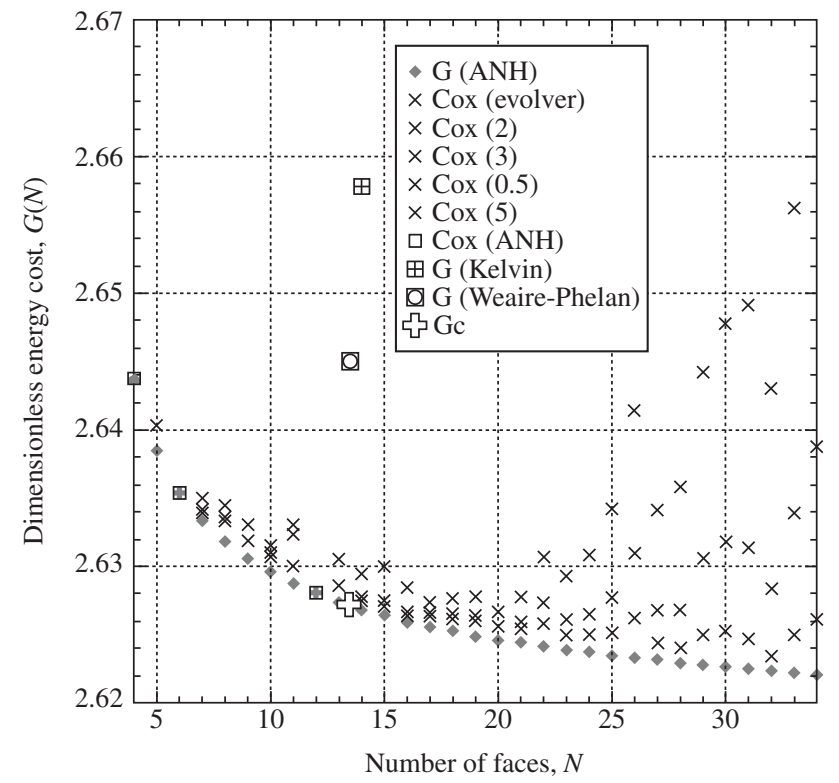

Figure 4. Dimensionless energy cost, $G, v s$. number of faces on a polyhedral cell, $N$. Data are shown for a narrow $(\approx 2.6 \%$ ) range of $G$-values for ANH's (black diamonds); for a selection of constructible irregular polyhedra and ANHs (crosses and open squares, respectively), as measured by Cox* using computer simulation; for Kelvin's orthic tetrakaidecahedron at $N=14$; for the Weaire-Phelan duplex tiling at $N=13.5$; and for the critical ANH at $N=13.397$. The G-values for highly symmetric ANHs form a lower bound for every class of polyhedra tested in various topological face combinations. The range of $G$-values exhibited by various polyhedra with a fixed number of faces increases with $N$, as many more topological combinations become possible.

* Cox SJ. Trinity College, Dublin, Ireland, Personal Communication; 2004. with the idea that ANHs provide an accurate representation or close 'proxies' of irregular polyhedra.

Of special significance is the dimensionless energy cost for the critical ANH,

$$
G(N)=\frac{1}{2} \frac{A(N)}{(V(N))^{\frac{2}{3}}}=2.62718 \ldots
$$

If one imagines a network constructed entirely of critical ANHs its dimensionless energy cost would be $\langle G\rangle=G_{c}$. The critical ANH value, 2.62718 , is lower than the dimensionless energy cost of tiling space with Kelvin's classical tetrakaidecahedron ${ }^{9}, \mathrm{G}=2.65737$, and with Weaire-Phelan's more recent duplex tiling ${ }^{10}, \mathrm{G}=2.64417$. The Weaire-Phelan tiling consists of two, irregular pentagonal dodecahedra and six tetrakaidecahedra. When combined into a unit cell, these eight polyhedra pack to form a duplex unit cell with the average value of $\langle N\rangle=13.5$ faces. Kelvin's and Weaire-Phelan's tiling are illustrated in Figure 5. The dimensionless energy cost of the critical ANH is significantly smaller than the others. Glicksman and Rios ${ }^{5}$ have recently shown that the critical ANH has the lowest possible dimensionless energy cost among other possibilities of tiling space.

\section{3D Analogue of von Neumann-Mullins Equation}

Polycrystalline networks are often subjected to some kind of processing involving heating to temperatures sufficiently high to assist the movement of the curved interfaces. As a result, grain growth takes place. During grain growth grains with convex faces, $N<N_{c}$, shrink and grains with $N>N_{c}$ grow.

The von Neumann-Mullins expression ${ }^{11,12}$, valid in two dimensions, for the growth/shrinkage of an individual grain, is well known for many years,

$$
\frac{d a}{d t}=M \gamma(n-6)
$$

where $a$ is the area and $n$ is the number of sides of a two-dimensional grain; $M$ and $\gamma$ - are the grain boundary mobility and grain boundary free energy per unit of length. Grains with less than six sides shrink, whereas grains with more than six sides grow.

An expression analogous to von Neumann-Mullins, but valid in three spatial dimensions, has been sought for over fifty years ${ }^{1,13-16}$. Such an expression may be obtained based on the assumption that a polycrystalline network can be represented, albeit abstractly, by

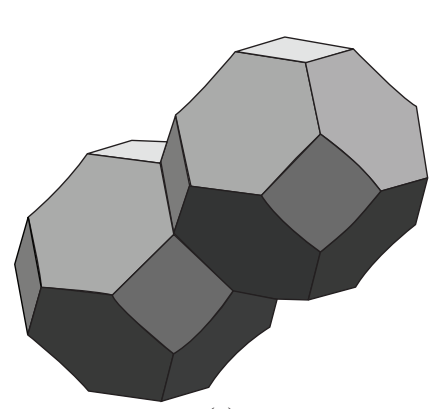

(a)

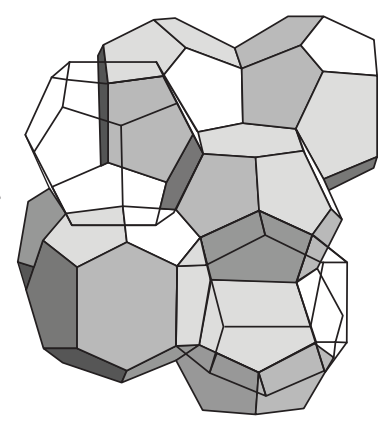

(b)
Figure 5. a) Kelvin's "relaxed" tetrakaidecahedra. Illustration suggests how the flatfaced version of these polyhedra would stack by translation to tessellate 3-d space and form a body-centered cubic (BCC) lattice. BCC lattice points would be located at the centroids of each Kelvin cell; and b) The WeairePhelan duplex tiling. This tiling consists of eight stacked polyhedra: two irregular pentagonal dodecahedra (shown as open frames)each isolated by six medial tetrakaidecahedra (shown as solid polyhedra). Figures adapted from http://www.susqu.edu/brakke/kelvin/kelvin.html and http://www.queenhill. demon.co.uk/polyhedra/wp/wp.htm. 
ANHs. This expression can be obtained by noticing that the interface velocity is given as:

$$
v=\frac{1}{A} \frac{d V}{d t}
$$

From Equations 6 and 19:

$$
\frac{d V}{d t}=-M \gamma H A
$$

Both $H$ and $A$ are known as a function of number of faces for the ANHs, Equations 5 and 9. Equation 20 can be put in a more convenient form, in which the rate of volume change depends only on the number of faces. This can be done noticing that:

$$
\frac{d V}{d t}=\frac{3}{2} V^{\frac{1}{3}} \frac{d V^{\frac{2}{3}}}{d t}
$$

so that:

$$
\frac{d V^{\frac{2}{3}}}{d t}=-\frac{2}{3} M \gamma A H V^{-\frac{1}{3}}
$$

In Equation 22 the scale factor, $\lambda$, cancels out and the right hand side is a function of the number of faces, only:

$$
\frac{d V^{\frac{2}{3}}}{d t}=-\left(\frac{128 \pi^{2}}{9}\right)^{\frac{1}{3}} M \gamma \frac{g(N)}{(f(N))^{\frac{1}{3}}}
$$

where $g(N)$ and $f(N)$ are defined above, Equations $9 \mathrm{~b}$ and $10 \mathrm{~b}$.

Equation 23 is the ANH-based 3-dimensional version of the von Neumann-Mullins equation. A plot of Equations 23 is shown in Figure 6 along with data from several computer simulations of evolving isotropic 3-dimensional networks. The agreement between the analytic theory and data from the simulations of Cox and Fortes? is excellent, especially where the data are for constructible ANHs. It is interesting that even the rate data shown in Figure 6, simulated from irregular isotropic networks, are in agreement with the present theory. The close correspondence between the growth rates predicted for ANHs and those found in simulations of irregular 3-dimensional networks is doubtless due to the robust character of the topological averages incorporated in the set of ANHs. In other words, ANHs act as accurate proxies for all the irregular polyhedra in a polycrystal exhibiting $N$ faces. Thus, they provide good estimates within each topological class for the average face curvature, surface area, and volume, all of which are relevant to determining the growth rate within each class of grains.

The dependency of the growth rate on the number of faces in 3D is a rather more complicated function than the elegant $(n-6)$ factor

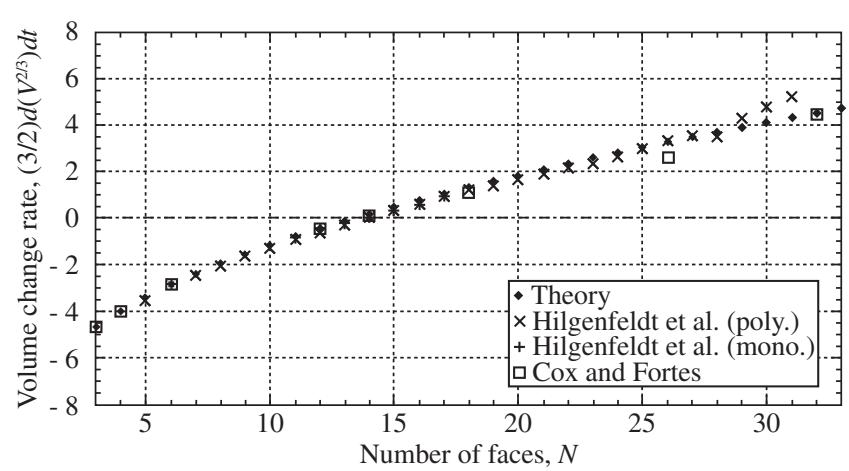

Figure 6. Comparison of the theoretical scale-free rates of volume change for various ANHs with Surface Evolver simulation data. The data from Cox and Fortes $^{7}$ are Surface Evolver simulations of isolated $N$-hedra, whereas the data from Hilgenfeldt et al. ${ }^{14}$ are based on Surface Evolver simulations of irregular isotropic network structures containing over 500 polyhedral cells. in 2D, derived in 1952 by von Neumann ${ }^{6}$. Simpler, linearized forms can be obtained for $3 \mathrm{D}$ by recognizing that $d V^{2 / 3} / d t$ itself is nearly linear in the variable $N^{1 / 2}$. Thus, one may write in lieu of the exact expression, Equation 23, the linear form:

$$
\frac{1}{M \gamma} \frac{d V^{\frac{2}{3}}}{d t}=A+B \sqrt{N}
$$

It is important to insure that the linearized approximation for $d V^{2 / 3} / d t$, Equation 24, vanishes at precisely the same value of $N$ where the exact expression, Equations 23 vanishes. Specifically, one can show that the root of Equation 23 occurs at the critical value, $N_{c}$. This constraint on Equation 24 may be applied by choosing the constant $\mathrm{A}$ in the form

$$
A=-B \sqrt{N_{c}}
$$

As a consequence of inserting Equations 25 into Equation 24, one is left with a linear kinetic equation for the growth of ANHs containing only a single parameter, $B$, viz.,

$$
\frac{1}{M \gamma} \frac{d V^{\frac{2}{3}}}{d t}=B\left(\sqrt{N}-\sqrt{N_{c}}\right)
$$

Fitting the one-parameter expression, Equation 26, to discrete kinetic data from the exact expression, Equation 23, over the interval from $3 \leq N \leq 49$ yields the result that $\mathrm{B} \approx 2.2709 \approx 9 / 4$, with an associated regression coefficient $\mathrm{R}=0.99991$. Figure 7 shows the discrete function $d V^{2 / 3} / d t$ plotted against $N^{1 / 2}$ together with the straight line representing Equation 26.Thus, one finds that the linear kinetic law in $3 \mathrm{D}$ for the scale-independent rate of volume change for ANHs is described as

$$
\frac{d V^{\frac{2}{3}}}{d t} \cong \frac{9}{4} M \gamma\left(\sqrt{N}-\sqrt{N_{c}}\right)
$$

Equations 26 or 27 are the 3D analogues to Equation 18 in 2D. The rate of area change vanishes in $2 \mathrm{D}$ for a polygon with six sides,

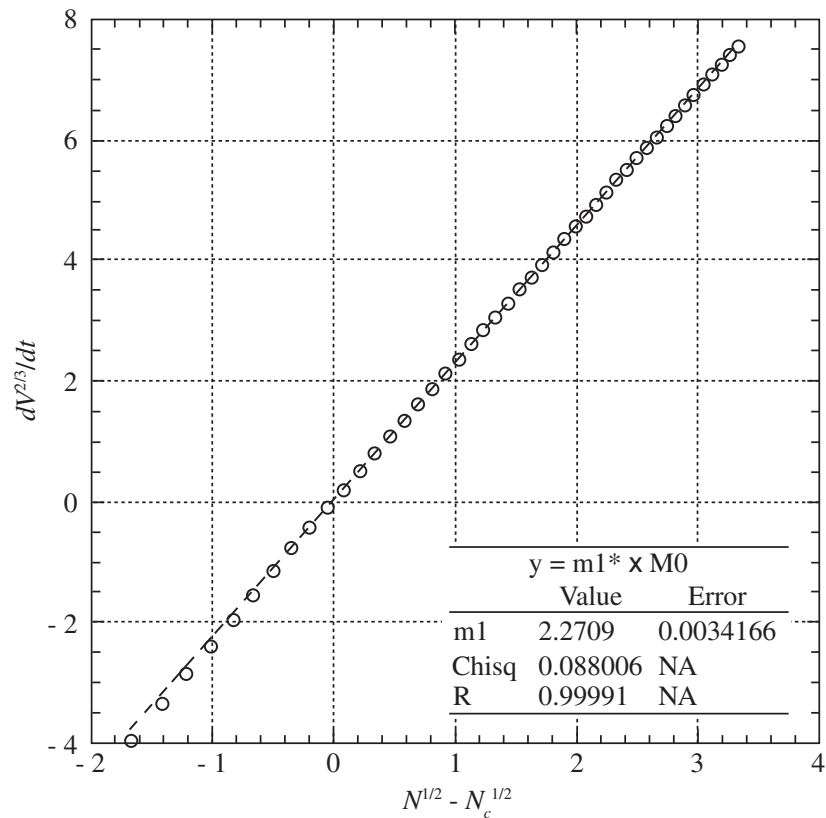

Figure 7. Linear regression of $(1 / M \gamma) / d V^{2 / 3} / d t v s$. $\left(N^{1 / 2}-N_{c}^{1 / 2}\right)$. There is good agreement between the exact values, Equation 23, and the straight line, Equation 26. 
whereas in $3 \mathrm{D}$ the rate of change of area and volume vanishes for the critical $\mathrm{ANH}$ with $\approx 13.39$ faces.

\section{Acknowledgments}

The authors are deeply indebted to Dr. Simon Cox, Physics Department, Trinity College, Dublin, Ireland, for sharing his Evolver simulation data with us. The authors express their appreciation for the financial support of this study derived from the John Tod Horton Distinguished Professorship in Materials Science and Engineering, at Rensselaer Polytechnic Institute. One of the authors (PRR) is grateful to the Conselho Nacional de Desenvolvimento Científico e Tecnológico, CNPq, and to the Fundação de Amparo à Pesquisa do Estado do Rio de Janeiro, FAPERJ, for his financial support.

\section{References}

1. Glicksman ME. Analysis of 3-D network structures. Philosophical Magazine. 2005; 85(1):3-33.

2. Glicksman ME. Energetics of polycrystals. Journal of Materials Science. 2005; 40(9-10):2149-2154.

3. Glicksman ME, Rios PR. Microstructural characteristics of 3-D networks. Zeitschrift für Metallkunde. 2005; 96(10):1099-1105.

4. Rios PR, Glicksman ME. Self-similar evolution of network structures. Acta Materialia. 2006; 54(4):1041-1051.

5. Glicksman ME, Rios PR. Minimal network partition using average ANH. Unpublished research, 2005.
6. Smith CS. Grain shapes and other metallurgical applications of topology. Metal Interfaces. Cleveland, OH: American Society for Metals; 1952. p. 65-109.

7. Cox SJ, Fortes MA. Properties of three-dimensional bubbles of constant mean Curvatura. Philosophical Magazine Letters. 2003; 83(4):281-287.

8. Brakke K. The motion of a surface by its mean curvature. Princeton: Princeton University Press; 1977.

9. Thomson W. (Lord Kelvin) On the division of space with minimum partitional area. Philosophical Magazine. 1887; 24(151):503-514.

10. Weaire D, Phelan R. A counterexample to Kelvin's conjecture on minimal surfaces. Philosophical Magazine Letters. 1994; 69(2):107-110.

11. Von Neumann J. Written discussion of grain shapes and other metallurgical applications of topology. Metal Interfaces. Cleveland, OH: American Society for Metals; 1952. p. 65-109.

12. Mullins WW. 2-dimensional motion of idealized grain boundaries. Journal of Applied Physics. 1956; 27(8):900-904.

13. Mullins WW. The statistical self-similarity hypothesis in grain-growth and particle coarsening. Journal of Applied Physics. 1986; 59(4):1341-1349.

14. Hilgenfeldt S, Kraynik AM, Koehler SA, Stone HA. An accurate von Neumann's law for three-dimensional foams. Physical Review Letters. 2001; 86(12):2685-2688.

15. Weaire D, Glazier JA. Relation between volume, number of faces and three-dimensional growth laws in coarsening cellular patterns. Philosophical Magazine Letters. 1993; 68(6):363-365.

16. Hilgenfeldt S, Kraynik AM, Reinelt DA, Sullivan JM. The structure of foam cells: isotropic Plateau polyhedra. Europhysics Letters. 2004; 67(3):484-490. 